\title{
Las plazas distritales de mercado en Bogotá: \\ una mirada de la inseguridad alimentaria y el consumo de alimentos en la localidad de Barrios Unidos*
}

\section{The district market \\ squares in Bogotá:}

a look at food insecurity and

food consumption in the

town of Barrios Unidos

\author{
Jhon Erick Gómez Moreno**
}

Recibido: 20 de Enero de 2018

Revisado: 25 de Febrero de 2018

Aprobado: 1 de Marzo de 2018

* El artículo hace parte del proyecto de investigación sobre las plazas de mercado en la ciudad de Bogota. Cómo citar este artículo: Gómez, J. E. (2019). Las plazas distritales de mercado en Bogotá: una mirada de la inseguridad alimentaria y el consumo de alimentos en la localidad de Barrios Unidos. Revista CIFE: Lecturas de Economía Social, 20(32), 139-166. doi: https://doi.org/10.15332/22484914.4859

** Economista de la Universidad Santo Tomás, especialista en Evaluación Social de Proyectos de la Universidad de Los Andes, magíster en Planeación para el Desarrollo de la Universidad Santo Tomás, Encargado de la gestión económica del proyecto 431 "Fortalecimiento del sistema distrital de plazas de mercado" del Instituto para la Economía Social - IPES. (2012 -2015). Investigador económico en el área de estudios socioeconómicos del Instituto para la Economía Social - IPES. Correo electrónico: jegomezm@ipes.gov.co 


\section{Resumen}

En Bogotá, el desarrollo comercial de las plazas de mercado constituye una oportunidad por la fuerte vigencia del mercado interno en la región central (la región Centro del país agrupa los departamentos de Tolima, Huila, Caquetá y Cundinamarca). El desarrollo de la conciencia ciudadana sobre la diversidad, el medio ambiente y el patrimonio cultural inmaterial de la gastronomía criolla, junto con los mercados campesinos, hacen pensar que las plazas pueden dar respuesta a las demandas institucionales de alimentos, contribuyendo con la seguridad alimentaria, reduciendo brechas económicas y precios, siendo un eje entre las demandas de sujetos y circuitos de la economía popular en los territorios de la ciudad. Por lo tanto, en este artículo se presenta un análisis económico, social y cultural de las plazas distritales de mercado de Bogotá, haciendo un énfasis en la localidad de Barrios Unidos. Además de un análisis y evaluación del proyecto 431 denominado "Fortalecimiento del sistema distrital de plazas de mercado". También haciendo énfasis en la inseguridad alimentaria de dicha localidad y su relación con el desarrollo humano.

Palabras clave: plazas de mercado, inseguridad alimentaria, consumo de alimentos, localidad Barrios Unidos.

Clasificación JEL: B55, J15

\section{Abstract}

In Bogota, the commercial development of the marketplaces, constitutes an opportunity for the strong force of the internal market in the central region (The central region of the country groups the departments of Tolima, Huila, Caquetá and Cundinamarca). The development of the civil conscience on the diversity and the environment and the cultural immaterial heritage of the creole gastronomy together with the rural markets, they make think that the squares can give response to the institutional demands of food, contributing with the food safety, reducing economic gaps and prices, being an axis between the demands of subjects and circuits of the popular economy in the territories of the city. Therefore in this article one presents an economic, social and cultural analysis of the distritales of marketplaces of Bogota, emphatically in the immersed ones in the Barrios Unidos village. Besides an analysis and evaluation of the project 431 called " strengthening of the system distrital of squares of market. Also doing emphasis in the food insecurity of the above mentioned village and his relation with the human development. Finally to present a measurement of the food consumption estimated for the year 2015 of the same one and his respective results.

Keywords: Marketplaces, food insecure, food consumption, Barrios Unidos village.

Classification JEL: B55, J15 


\section{Introducción}

Este artículo de investigación presenta las reflexiones de los comerciantes y visitantes de las plazas distritales de mercado, especialmente, en lo relacionado al consumo de alimentos no procesados en la ciudad de Bogotá; para esto, se recogen los resultados de la investigación sobre el consumo de alimentos en la localidad de Barrios Unidos, a través de la elaboración de un modelo ${ }^{1}$ con datos secundarios.

Localidad ubicada en el norte de Bogotá, representa el 1.4\% del área total de la ciudad, es la decimoquinta localidad en extensión territorial (1.190 hectáreas); no posee suelo rural, ni suelo de expansión. Limita por el norte con la localidad de Suba; al sur con la localidad de Teusaquillo; al oriente con la localidad de Chapinero y por el occidente con la localidad de Engativá. Por su posición geográfica, la localidad es considerada como una localidad de conexión con los cuatro puntos geográficos de la ciudad y la región, a través de la autopista Norte y la avenida Calle 72. (Cámara de Comercio de Bogotá, 2007, p. 17)

Teniendo como referente los equipamientos de abastecimiento de alimentos como las plazas distritales del Doce de Octubre y Siete de Agosto; es relevante reconocer cómo estos sitios han sido catalizadores urbanos, donde se median prácticas culturales, por ello, la categoría central de este escrito son los espacios denominados plazas de mercados.

Por ende, dichas plazas de mercado son lugares destinados para la prestación de un servicio al público, con la misión de garantizar la oferta de productos básicos, principalmente de origen agropecuario, de consumo doméstico, garantizando condiciones de libre competencia para satisfacer las necesidades de todas y cada una de las familias que integran la comunidad en general, en condiciones óptimas de carácter ambiental, sanitario, de seguridad, de calidad, eficiencia y economía dentro de un libre mercado. De tal manera estos espacios "en las ciudades del mundo han sido al mismo tiempo foros y espacios de comercio de bienes básicos, y como tales están ligadas a las identidades urbanas que se han forjado en muchas ocasiones desde sus propias entrañas" (Russo, 2011, p. 1).

En tanto, de las 64 plazas de mercado existentes en la ciudad de Bogotá, 45 cuentan con administraciones comunitarias, 19 son públicas y están a cargo del Instituto para la Economía Social (IPES), como entidad adscrita a la Secretaria de Desarrollo Económico con personería jurídica, autonomía administrativa y patrimonio propio, según el Acuerdo 257 de 2006 (Correa, 2014).

En su conjunto estas 19 plazas de mercado conforman el Sistema Distrital de Plazas de Mercado (SDPM), ubicadas en 13 de las 20 localidades de la ciudad. Como se muestra a continuación:

1 En el marco de la maestría en Planeación para el Desarrollo se realizó una investigación que buscaba determinar el consumo de alimentos no procesados en la localidad de Barrios Unidos, a través de dos estudios de caso: las plazas de mercado de los barrios Siete de Agosto y Doce de Octubre, a partir de un modelo económico simple. 
Figura 1. Ubicación de las Plazas Distritales de Mercado en las localidades de Bogotá.

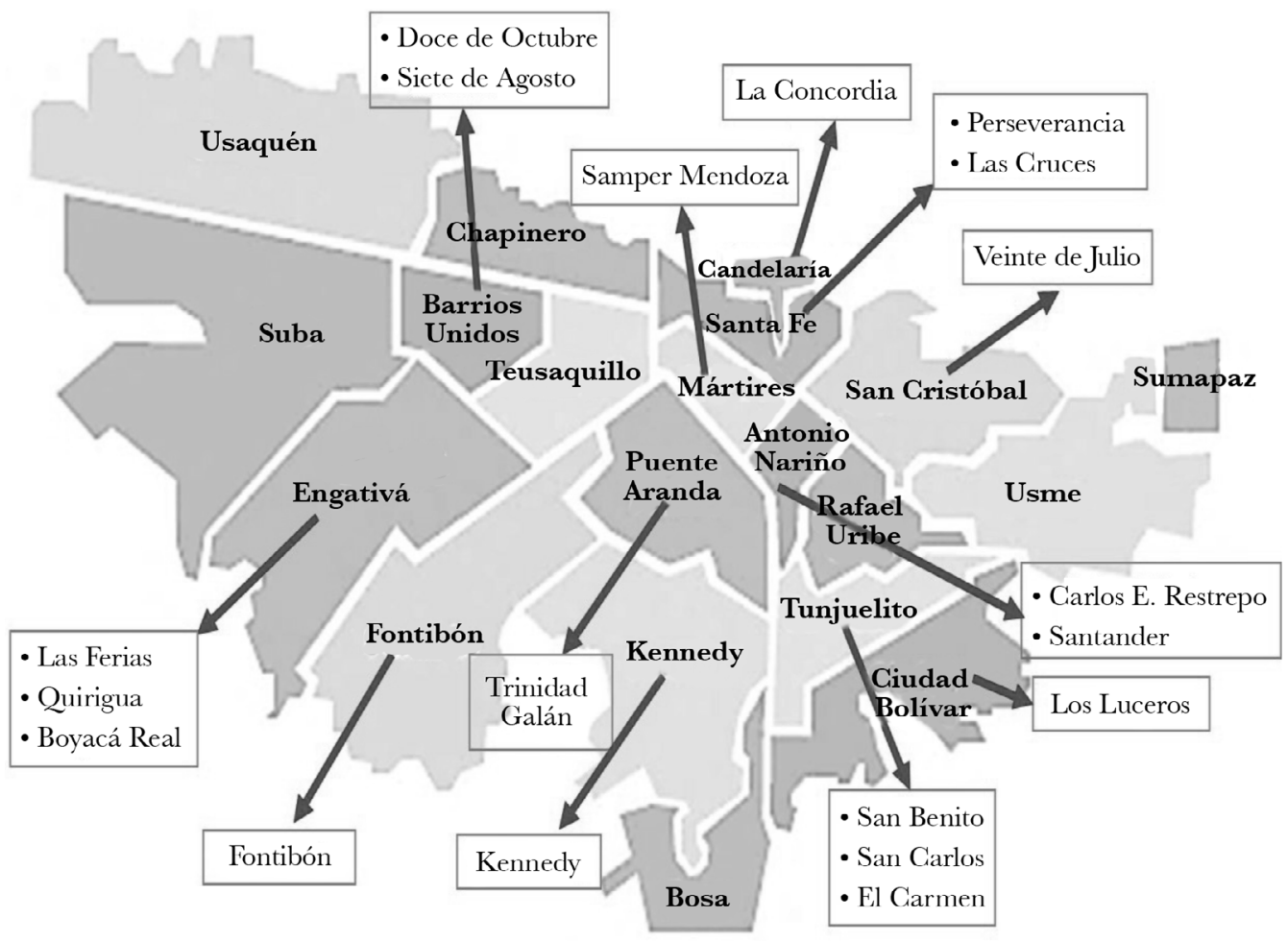

Fuente. Subdirección de Emprendimiento Servicios Empresariales y Comercialización, Sistema Distrital de Plazas de Mercado, 2014

Reconocer la tradicionalidad de estos espacios no solo sirve para estrechar los lazos difíciles entre el campo y la ciudad, entre el producto y el consumidor. Es también la forma de revelar el proceso de las cosas, de mostrar su procedencia, su trato, su preparación. También los rostros humildes que cultivan los frutos, que los ven crecer (Instituto Distrital de Turismo (IDT), Instituto para la Economia Social (IPES) y Fundación Escuela Taller (FET), 2013).

Estos atributos y otros que se observaron en la investigación que hace parte de la caracterización de las plazas de mercado, la relación de los sujetos o actores de estas y el consumo de alimentos no procesados de la localidad de Barrios Unidos, hacen necesario realizar una investigación de enfoque mixto y de tipo explicativo, basada en mediciones cuantitativas y cualitativas respectivamente. Utilizando la revisión de fuentes secundarias como el proyecto 431 del Instituto para la Economía Social (IPES), denominado "Fortalecimiento del sistema distrital de plazas de mercado", así como el informe de Gestión del Director del IPES en 2015 y documentos de las Secretarias de Desarrollo Económico y de Hacienda respectivamente, durante el periodo de estudio 
de la investigación, es decir, entre 2012 y 2015; además de la acción participante en las plazas de mercado distritales de los barrios Siete de Agosto y Doce de Octubre, ubicados en la localidad de Barrios Unidos; este artículo presenta la caracterización de las plazas distritales de mercado en mención y su influencia en el consumo de alimentos de la localidad, de la cual hacen parte importante como centro de comercialización y distribución de alimentos, contribuyendo a la seguridad y soberanía alimentaria de los habitantes de la localidad.

El periodo de análisis fue entre 2012 y 2015, duración del plan de desarrollo Bogotá Humana, en donde el Instituto para la Economía Social (IPES), ha tenido a su cargo la administración de las 19 plazas, y fue el abanderado del proyecto 431 denominado "Fortalecimiento del sistema distrital de plazas de mercado", que hace parte del programa de "Soberanía y seguridad alimentaria y nutricional". La soberanía alimentaria en el marco de la Bogotá Humana, entendida como poder y capacidad de un territorio o una comunidad para decidir qué, cómo, cuándo y cuánto produce y consume de alimentos; ello supone hacer efectivos los conceptos de seguridad alimentaria de un pueblo, una vecindad o una comunidad, que incluyen diversos elementos aportados por instituciones y organizaciones sociales (Secretaría de Desarrollo Económico, 2012).

Para ello, se procedió a hacer un análisis cualitativo, teniendo presente las potencialidades de las dos plazas estudiadas, las sugerencias por parte de los visitantes y encargados de las mismas para el mejoramiento de su dinámica comercial, incluyendo la caracterización de su capacidad instalada y sectores económicos que las conforman.

El instrumento utilizado fue la observación participante -implica tener en cuenta la existencia del observador, su subjetividad y reciprocidad en el acto de observar (Hernández, Baptista y Fernández, 2010)-, durante los años 2012-2015, sin embargo, la sistematización fue únicamente del 2015, adicional, se realizó un análisis documental del proyecto 431 denominado "Fortalecimiento del sistema distrital de plazas de mercado", del Instituto para la Economía Social (IPES), el cual busca fortalecer los vínculos existentes entre las plazas de mercado y el sistema de redes de abastecimiento de la ciudad, en la perspectiva de contribuir con la seguridad alimentaria y rendición de cuentas del IPES, con el fin de construir un modelo para determinar el consumo de alimentos en la localidad de Barrios Unidos.

Teniendo en cuenta lo anterior, en relación con lo correspondiente al análisis cuantitativo, se analizó el grado de inseguridad alimentaria en la localidad, teniendo presente la dimensión de seguridad alimentaria en la evaluación del desarrollo humano, y el modelo planteado por el autor en donde se tuvo en cuenta cambios tales como: incremento de la población y el incremento de los precios entre el año 2004 y 2015, esto con el fin de tomar como referencia el estudio del 2003 del Instituto Latinoamericano de Servicios Legales Alternativos (ILSA), denominado "Consumo de alimentos en Bogotá, 
déficit y canasta básica recomendada" (Yepes y Suárez, 2005) y traerlo a valor presente. Con estos resultados se buscó tener una idea cercana del comportamiento del consumo de alimentos a nivel monetario de la localidad de Barrios Unidos al final del plan de desarrollo Bogotá Humana, lo que permitió en la investigación evidenciar el tema de consumo de alimentos en la localidad. Adicional, se realizaron algunas entrevistas para corroborar lo planteado en dicho modelo y determinar si las plazas distritales de mercado de la localidad de Barrios Unidos suplen la demanda de alimentos de los habitantes, y si se contribuye a garantizar la seguridad alimentaria de esta.

La metodología permitió que con los atributos descritos se reconocieran las relaciones económicas y sociales alrededor de la comercialización de productos en las plazas de mercado, las cuales han venido perdiendo su tradicionalidad y cada vez son menos visitadas por la población vecina.

Ya que hoy ese antiguo espacio denominado plaza de mercado, en principio de localización del comercio, escenario de los procesos revolucionarios, se debate en la lucha por permanecer ante nuevas prácticas y discursos del mercado y de la cultura como son las grandes superficies o hipermercados y centros comerciales (Castiblanco, 2011).

Este artículo se desarrolla en cinco grandes secciones. En la primera parte se presenta todo lo relacionado con las plazas distritales de mercado y una descripción de las plazas del Doce de Octubre y Siete de Agosto, a nivel económico, social, cultural y administrativo. En la segunda, se presenta y analiza el proyecto 431 denominado "Fortalecimiento del sistema distrital de plazas de mercado" y una breve evaluación de los resultados de este. En la tercera, se hace un análisis acerca de la inseguridad alimentaria en la localidad de Barrios Unidos y su relación con el desarrollo humano. En la cuarta, se presentan los resultados de la medición del consumo de alimentos estimado para el año 2015, a través del modelo propuesto por el autor en la localidad y sus respectivos resultados. Por último, se presentan las conclusiones.

\subsection{Un encuentro con las plazas de mercado de la localidad de Barrios Unidos: Doce de Octubre y Siete de Agosto}

La plaza de mercado puede ser vista como un microcosmos colorido, la cual ha sido estudiada por diferentes disciplinas como la economía, la sociología, la antropología, entre otras, en donde todas estas disciplinas confluyen en que son lugares tradicionales destinados para la prestación de un servicio al público, con la misión de garantizar la oferta de productos básicos, principalmente de origen agropecuario, de consumo doméstico, garantizando condiciones de libre competencia para satisfacer las necesidades de todas y cada una de las familias que integran la comunidad en general, en condiciones óptimas de carácter ambiental, sanitario, de seguridad, de calidad, eficiencia y economía dentro de un libre mercado (Instituto Distrital de Turismo (IDT) et al., 2013). 
Estas han funcionado en los últimos años sin mayores transformaciones, condición que ha conducido a una pérdida de sus dinamismos, lo cual se ve reflejado en el bajo impacto en el aprovisionamiento de alimentos básicos en la ciudad de Bogotá, unido al deterioro físico que se hace notorio y que conlleva al incumplimiento de las exigencias sanitario-ambientales (IPES, 2012).

De otra parte, el Distrito Capital destina cada vez menos recursos para el funcionamiento de las plazas, puesto que estos no se ejecutan en su totalidad, lo cual se denota en la siguiente tabla elaborada por el área de presupuesto del IPES, según información suministrada por el Presupuesto Distrital (Predis) de la Secretaria de Hacienda Distrital.

Tabla 1. Presupuesto asignado al proyecto 431 "Fortalecimiento del sistema distrital de plazas de mercado", Bogotá Humana (2012-2015)

\begin{tabular}{|c|cccc|}
\hline \multicolumn{4}{|c}{ Proyecto 431 "Fortalecimiento del sistema distrital de plazas de mercado" } \\
\hline Vigencia & Plurianual & Asignado & Ejecutado & \% Ejecución \\
\hline 2012 & $\$ 4.469$ & $\$ 4.469$ & $\$ 4.385$ & $98.12 \%$ \\
\hline 2013 & $\$ 10.000$ & $\$ 9.391$ & $\$ 7.707$ & $82.07 \%$ \\
\hline 2014 & $\$ 16.070$ & $\$ 14.107$ & $\$ 13.286$ & $94.18 \%$ \\
\hline 2015 & $\$ 16.070$ & $\$ 12.909$ & $\$ 9.086$ & $70.39 \%$ \\
\hline Total & $\$ 46.609$ & $\$ \mathbf{4 0 . 8 7 6}$ & $\$ \mathbf{3 4 . 4 6 4}$ & $\mathbf{8 4 . 3 1 \%}$ \\
\hline
\end{tabular}

Fuente. Predis, Secretaría de Hacienda Distrital. Septiembre de 2015

Como se observa en la tabla anterior con relación a la ejecución presupuestal en 2015 (Secretaría de Hacienda Distrital, 2015) fue indicada como el 70.39 \%, que de acuerdo a estándares fiscales fue una ejecución relativamente baja, si esta es comparada con la programación inicial del proyecto respecto al plan plurianual de inversión, dicha ejecución fue del $56.5 \%$, lo cual impacta significativamente el cumplimiento de las metas propuestas por el proyecto formulado.

Esta condición conlleva a la necesidad de replantear la forma como se han venido interviniendo sus prioridades, sus condiciones comerciales, sociales, de visibilización, de priorización de lo público, pero en especial, para hacer más eficiente el manejo de los escasos recursos que permitan atender las exigencias higiénicas sanitarias y hacerlo en la mayor cantidad de las 19 plazas públicas.

En cuanto a la actualización de información acerca de las plazas públicas de la ciudad, no se cuenta con datos actualizados que faciliten, entre otros aspectos, la toma de 
decisiones, el manejo de variables para implementar las políticas ya definidas y las prioridades a atender, en donde se priorice la participación de los actores que están en las plazas, es decir, de los comerciantes, de los usuarios y de otras instituciones.

En este sentido, es necesario adelantar una caracterización y análisis de información de las plazas de mercado y así conocer la realidad de cada una, el territorio en donde se encuentra, la importancia de los circuitos económicos que se han generado a su alrededor, los servicios que requieren los usuarios, las realidades comerciales.

La realización de esta caracterización tiene su soporte en lo contenido en el Plan de Desarrollo Bogotá Humana, el eje estratégico "Una ciudad que supera la segregación y la discriminación: el ser humano en el centro de las preocupaciones del desarrollo" y el programa de "Soberanía y seguridad alimentaria" en la estrategia misional del IPES, que prioriza la atención a la población de plazas de mercado, así como en la estructuración de uno de los ejes de intervención de estas, que corresponde a la gestión económica; con esto se podrían facilitar los procesos de planeación y la programación de las inversiones en las plazas.

Lo anterior con el fin de establecer las condiciones económico-espaciales de las plazas de mercado, con el propósito de aplicar los programas de apoyo y fortalecimiento; reconociendo sus condiciones, ventajas comparativas y posibilidades de desarrollo.

\subsection{Una descripción de la plaza de mercado Doce de Octubre}

A continuación, se hará una descripción de la tradicional plaza mercado de mercado 12 de Octubre, ubicada en la calle 72 \# 51-62. Está situada en el corazón de Bogotá, más exactamente en el barrio que lleva su mismo nombre, en la localidad de Barrios Unidos. Fue construida en el año 1952 por la Empresa Distrital de Servicios Públicos "EDIS", que a su vez cedió la plaza a la Procuraduría de Bienes del Distrito Capital en 1996. Antes de su construcción, estaba cercada por latas y su piso era de tierra (IPES, 2015).

Sus fundadores, comerciantes que iniciaron cuando la plaza era algo así como un potrero, narran anécdotas y costumbres de la época, como por ejemplo lo citando en una entrevista realizada a uno de los comerciantes del Comité de Desarrollo Integral de la plaza Doce de Octubre, en septiembre de 2015:

Las básculas que se usaban era en un lado una pesa y en el otro el canasto con el mercado que se deseaba pesar; se hacían regalos entre ellos el día de amor y amistad, realizaban corridas de toros y celebraban la fiesta de la Virgen del Carmen, actividad que aún permanece. (Entrevista 1)

Es famosa por la variedad en pescados y lácteos y la fritanga de doña Segunda. Tiene un puesto de lectura, en el que se realizan actividades lúdicas y talleres de lectura para los 
niños de los visitantes. Atraídos por la lechona, a esta plaza confluyen personajes de la vida política de nuestro país y extranjeros que desean probar este producto (IPES, 2015).

A partir de la observación participante en la plaza de mercado se pudo determinar que esta tenía una capacidad instalada de 311 puestos de mercado, para diciembre de 2015 contaba con 147 comerciantes, es decir, tenía una ocupación del 47 \%, en donde la distribución de estos por cantidad de oferentes de cada una de las actividades económicas que se desarrollan en la plaza se presenta a continuación:

Figura 2. Capacidad instalada plaza distrital de mercado Doce de Octubre

\section{Plaza de Mercado Doce de Octubre}

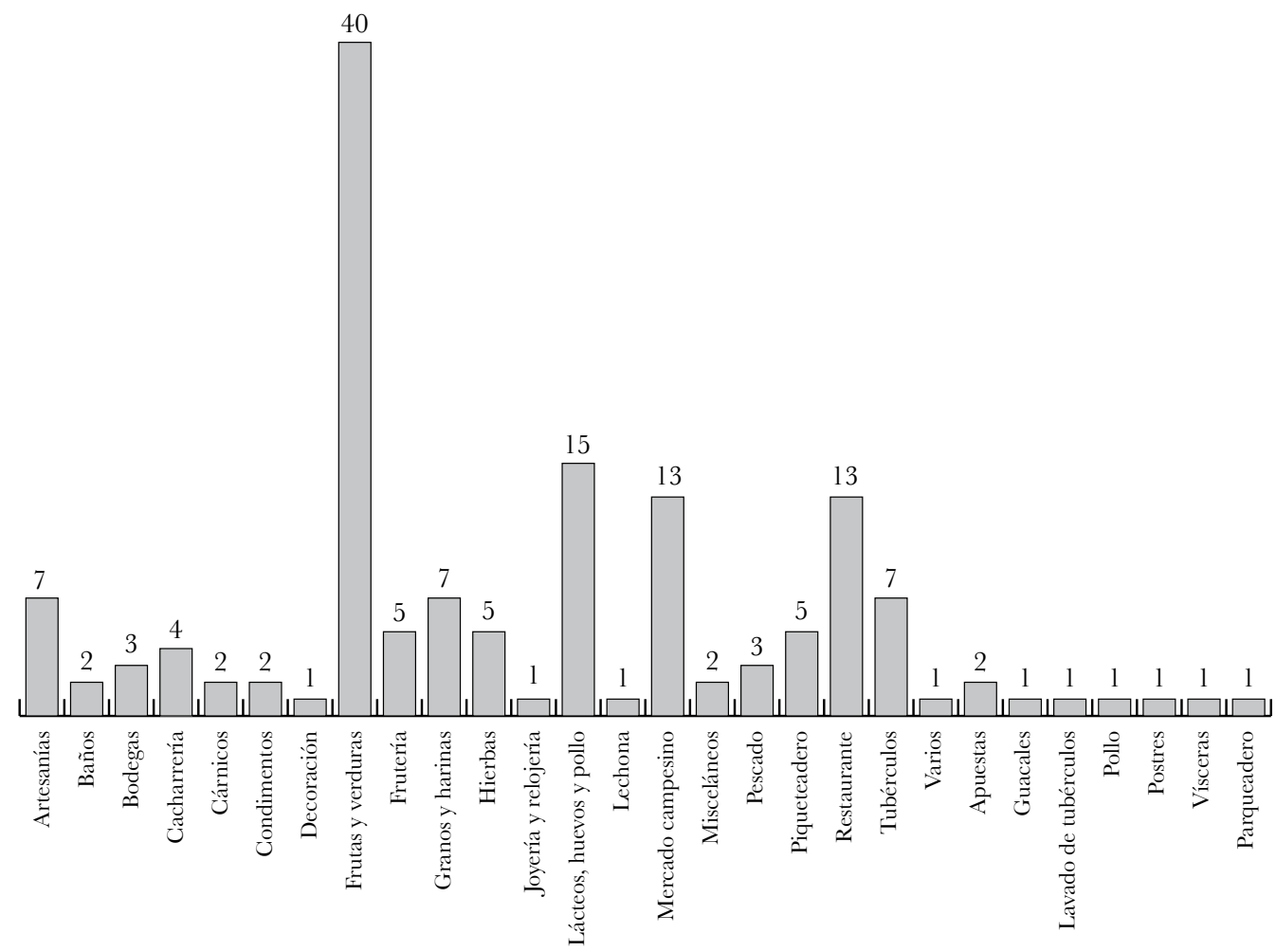

Fuente. Elaboración propia con base en el censo de comerciantes por actividad económica según información de la administración de la plaza, 2015

Cuenta con su propia dinámica comercial, en donde las actividades económicas denominadas como su "fuerte" son los restaurantes, la venta de lácteos y cárnicos principalmente. $\mathrm{Al}$ preguntarle a la administradora de la plaza de mercado sobre las sugerencias que tenía acerca del mejoramiento de la dinámica comercial, afirmó que: 
Para contestar este punto se consideraron cuatro aspectos, los cuales de alguna manera resumen lo que a juicio es de gran importancia para mejorar y en consecuencia incrementar el comercio en la plaza:

El edificio: en el estado actual de la plaza del Doce de Octubre se requiere un cambio extremo tanto en partes de la estructura (techos, pisos, puestos entre otros), como en su parte externa (específicamente zona sur).

Los comerciantes: otro cambio importante en el aspecto de imagen está relacionado con los comerciantes, a quienes se les proporcione formación y capacitación en diversos temas, tales como: atención a clientes, manejo de productos y lo relacionado con el manejo del negocio (emprendimiento y administración).

Ocupación: al mostrar una imagen más amigable en la plaza, es factible que más personas quieran hacer parte de este trabajo, y con una labor de divulgación incluso de los comerciantes que ya pertenecen a esta plaza se lograría un mayor porcentaje de ocupación.

Eventos y promociones: en articulación con otras entidades tales como alcaldías locales, bomberos, movilidad, JAL, programar y realizar jornadas culturales, artísticas, de salud, de capacitación, entre otras; dirigidas a clientes, comerciantes y visitantes de la plaza. Aprovechando la asistencia y participación de visitantes (clientes) realizar promoción de productos, descuentos, en general actividades que generen mayor venta.

Otro punto relacionado con las ventas es tener "días especiales", donde se promocione un producto o una de las actividades, ofreciendo mejores precios en el producto o la actividad promocionada.

En conclusión, este lugar es un referente de prácticas ancestrales y culturales de gastronomía para muchos bogotanos, por tanto, es un patrimonio vivo, porque pasó de ser una plaza de barrio o localidad a un referente de todo el distrito.

\subsection{Una descripción de la plaza de mercado Siete de Agosto}

A continuación, se hará una descripción de la tradicional plaza mercado de mercado Siete de Agosto, ubicada en la calle 66 \# 23-20. La cual a finales de los años 50, funcionaba en una rotonda central donde se vendían granos, frutas, verduras y líchigo, en un terreno donado por la familia del señor Nemesio Camacho, quien también había facilitado el predio donde hoy funciona el estadio El Campin (Entrevista 2, realizada a uno de los comerciantes que lleva más de cincuenta años en la plaza de mercado del Siete de Agosto, en octubre de 2015).

El mercado de la plaza provenía de la región cundiboyacense, con el atenuante que en este mismo sector salían y llegaban las flotas procedentes de Cundinamarca, Boyacá y Santander. Inicialmente, se realizaba la comercialización en unas casetas o chazas improvisadas que los mismos comerciantes construyeron para tal fin. En 1972 se hace 
la entrega oficial a los comerciantes de la nueva plaza, la cual se conserva con pocos cambios a la fecha (IPES, 2015).

A partir de la observación participante en la plaza de mercado se pudo determinar que esta contaba con 275 módulos de comerciantes de diferentes actividades económicas, destacándose las frutas, verduras, cárnicos, pescado, vísceras, lácteos, fruterías, papa, plátano, aguacate, artesanías, graneros, ropa, calzado y la sección de restaurantes. Distribuidos de la siguiente manera:

Figura 3. Capacidad instalada plaza distrital de mercado Siete de Agosto

\section{Plaza de Mercado Siete de Agosto}

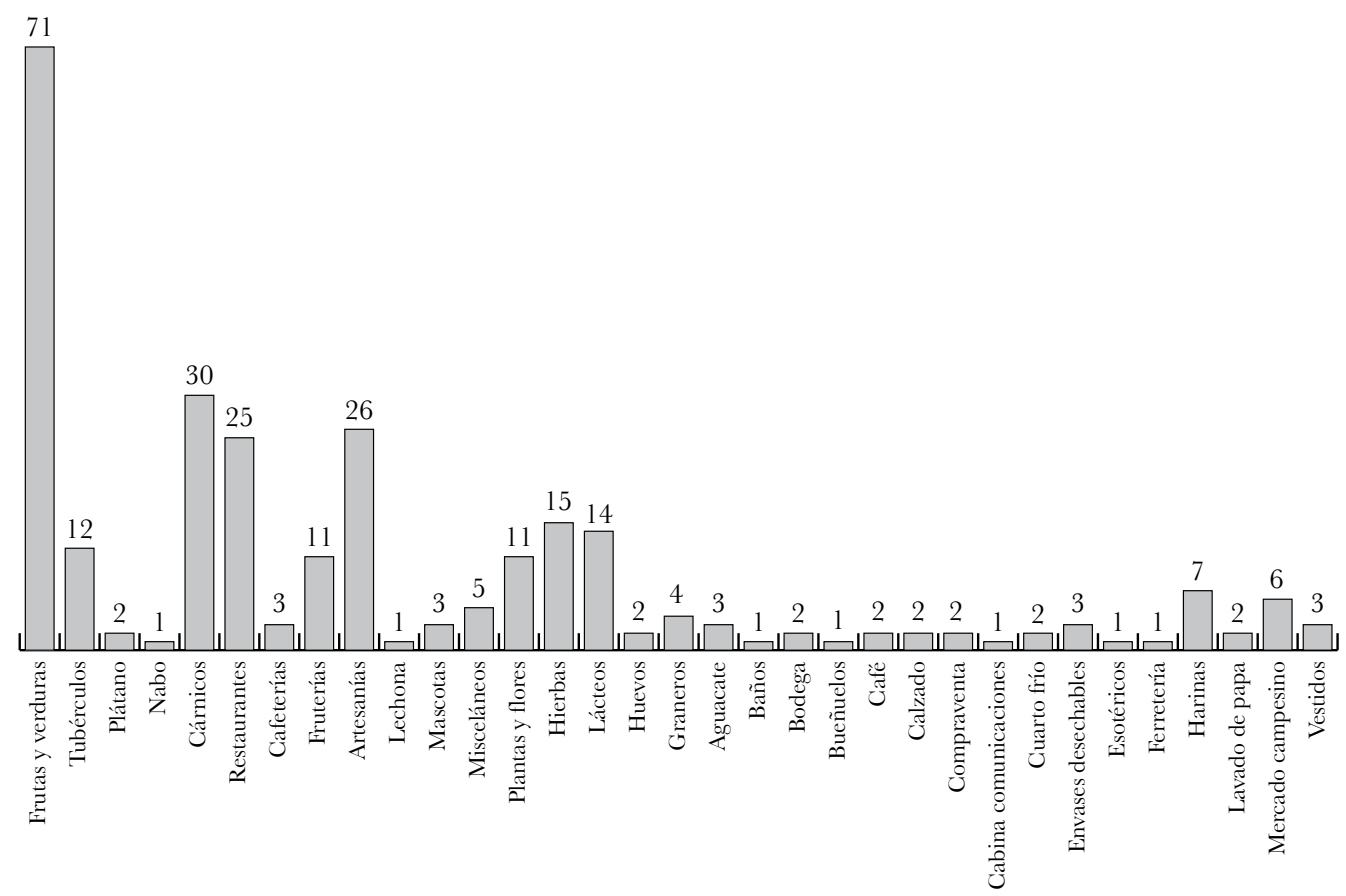

Fuente. Elaboración propia con base en el censo de comerciantes por actividad económica según información de la administración de la plaza

Cuenta con una dinámica comercial propia, en donde las actividades económicas denominadas como su "fuerte" son los restaurantes, las fruterías y la venta de artesanías principalmente. Al preguntarle al administrador de la plaza de mercado sobre las sugerencias que tenía acerca del mejoramiento de la dinámica comercial, afirmó que: 


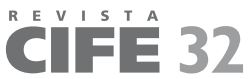

Revista CIFE / ISSN: 0124-3551 e-ISSN: 2248-4914 / Bogotá-Colombia / Vol. 20 N.o 32 / enero-junio 2018 / pp. $139-166$

- A la plaza le falta el parqueadero, ya que no hay espacio donde el cliente pueda dejar su vehículo. Además de pintarla en la parte exterior para generar visibilidad.

- Falta un mapa de ubicación, con el fin de orientar al cliente.

- Modernización de los espacios para poder ubicar mejor los puestos, ya que la plaza esta sobresaturada (116\%), afectando los espacios comunes y corredores.

- Capacitaciones, charlas para los comerciantes de la plaza con prácticas para mejorar la atención al cliente.

- Invertir en el arreglo del cuarto de residuos, lo anterior teniendo en cuenta que es muy pequeño para la demanda, se encuentra en muy mal estado (requerimiento también de la Secretaria de Salud) y por encontrarse en la parte exterior le da muy mal aspecto.

- Reforzar toda la cubierta de la plaza, ya que constantemente las tejas se caen y se puede generar algún riesgo para el cliente, el comerciante y sus productos.

- Revisión en instalaciones eléctricas, ya que constantemente se saltan los tacos generando daño en los equipos eléctricos.

- Mejorar la visibilidad de la plaza, con la reparación de las lámparas de luz en los pasillos principales.

- Lograr uniformidad, mediante una plazoleta de comidas (uniformes, manteles y mesas).

- Proponer unificación de uniformes y un logo para los comerciantes y dependientes, dando así empoderamiento de la plaza.

Gracias al trabajo de campo se puede evidenciar que la antigüedad de la plaza en el sector le ha permitido su reconocimiento y buen nombre. Además, que la presentación de un mercado integral compuesto por frutas, verduras, artesanías, cárnicos, restaurantes, lácteos, cafeterías, entre otros, ofrece una amplia gama de productos para el público que visita la plaza. Además de una excelente ubicación, ya que se tiene acceso a esta por vías importantes de la ciudad y cuenta con zonas muy comerciales que estimulan su visita.

Además, la calidad en los productos que se ofrece al público en cuanto a limpieza y transparencia en la venta garantiza la seguridad alimentaria de los visitantes, e influye en la satisfacción del cliente. 


\section{El fortalecimiento de las plazas distritales de mercado}

La política distrital de seguridad alimentaria y nutricional 2007-2015, establece como objetivo general:

Garantizar de manera progresiva, estable y sostenible las condiciones necesarias para la seguridad alimentaria y nutricional de la población del Distrito Capital, en perspectiva de ciudad-región. Para su desarrollo se planteó como ejes estratégicos de acción pública: la disponibilidad de alimentos suficientes, nutricional y culturalmente adecuados e inocuos y de agua en la cantidad mínima esencial y con la regularidad y sostenibilidad necesarias para el consumo humano; el acceso de toda la población a los alimentos y al agua potable en forma autónoma y en igualdad de condiciones y oportunidades; y prácticas de alimentación y modos de vida saludables en el marco de la diversidad cultural nutrición y entornos saludables. (Decreto 508, 2007, p. 8)

En tal sentido, corresponde al Estado garantizar un entorno seguro para el ejercicio del derecho a la alimentación, respetar y promover las condiciones para que los individuos puedan acceder, por sí mismos, a los alimentos, en la cantidad y la calidad requeridas.

Por tal motivo, la Alcaldía Mayor de Bogotá en su plan de desarrollo Bogotá Humana (2012-2016), en su eje estratégico "Una ciudad que supera la segregación y la discriminación: el ser humano en el centro de la preocupación del desarrollo", dentro del programa: "Soberanía y seguridad alimentaria y nutricional"; por medio del Instituto para la Economía Social (IPES), entidad adscrita a la Secretaría de Desarrollo Económico, formuló el proyecto 431, denominado "Fortalecimiento del sistema distrital de plazas de mercado".

El objeto general que se propuso cumplir con este proyecto fue el de consolidar el sistema distrital de plazas de mercado, para mejorar su competitividad a fin de contribuir con la seguridad alimentaria, reducir las brechas económicas y disminuir los precios. Este objetivo se encuentra asociado a la política la política de seguridad alimentaria, la cual es definida de la siguiente manera:

Seguridad alimentaria y nutricional es la disponibilidad suficiente y estable de alimentos, el acceso y el consumo oportuno y permanente de los mismos en cantidad, calidad e inocuidad por parte de todas las personas, bajo condiciones que permitan su adecuada utilización biológica, para llevar una vida saludable y activa. (CONPES 113, 2008, p. 1)

Lo anterior, especialmente en los componentes de acceso y disponibilidad de alimentos inocuos.

La posibilidad de acceso en relación con la reducción de brechas económicas y la disminución de los precios propuesta en el objetivo general demanda una visión regional de la productividad agropecuaria, el acortamiento de las cadenas de comercialización para los comerciantes de las plazas, y de estos con los tenderos y ciudadanos consumidores en 
general. Esta previsión parece haber sido considerada por la Secretaría de Planeación Distrital y la Región Administrativa Especial de Planeación (Rape) que integra los departamentos de Boyacá, Cundinamarca, Meta, Tolima y la ciudad de Bogotá, quienes han definido la soberanía y la seguridad alimentaria como una de las líneas estratégicas del desarrollo regional (Rape, s.f.).

Otro aspecto fundamental en la articulación de las plazas de mercado bogotanas a la política de seguridad alimentaria es que representan equipamientos fundamentales para la distribución de alimentos, articulación de productores rurales y consumidores locales (Decreto 315, 2006).

No obstante, los objetivos específicos del proyecto se encuentran asociados a aspectos como implementación de planes de manejo de residuos sólidos, cumplimiento de normas de sismo resistencia, el rediseño del modelo de gestión administrativa de las plazas y la articulación de las 19 plazas de mercado al sistema de redes de abastecimiento de la ciudad (IPES, 2012); este último limitado de manera importante en su aplicación por la concentración de actividades de carácter interno de las plazas, principalmente administrativas.

Puede inferirse entonces que, si bien el proyecto se enmarca en la política seguridad alimentaria, sus objetivos específicos no contribuyen adecuadamente al cumplimiento tanto del objetivo general del proyecto, como de las orientaciones de política a las que están evocadas las plazas por su naturaleza. Para el logro de tales objetivos se implementaron tres elementos dinamizadores que posibilitaron fortalecer los procesos mencionados (IPES, 2012).

Inicialmente la gobernanza, que es la realización de relaciones políticas entre diversos actores involucrados en el proceso de decidir, ejecutar y evaluar decisiones sobre asuntos de interés público, proceso que puede ser caracterizado como un juego de poder, en el cual competencia y cooperación coexisten como reglas posibles; y que incluye instituciones tanto formales como informales. La forma e interacción entre los diversos actores refleja la calidad del sistema y afecta a cada uno de sus componentes; así como al sistema como totalidad (Whittingham, 2010).

Gobernanza: este elemento buscaba fortalecer la administración y manejo de las 19 plazas de mercado entre la institución, los comerciantes y usuarios, a través de la participación ciudadana, las alianzas, la revalorización cultural de las plazas y la comunicación para transformar los conflictos y recuperar el sentido de lo público en defensa de los bienes comunes.

Gestión económica: buscaba dinamizar la recuperación comercial y el fortalecimiento económico en las 19 plazas de mercado alrededor de la canasta familiar básica en clave de seguridad y soberanía alimentaria. 
Mantenimiento, adecuación e infraestructura: esta estrategia buscaba estructurar diseños y rediseños de adecuación y mantenimiento de la planta física, la dotación, el mobiliario y el ordenamiento de la plaza como espacio público, de acuerdo con la factibilidad de cada plaza, el PMR, los POT y el escenario de oportunidades en el entorno.

Los actores implicados en el desarrollo del proyecto fueron:

- Campesinos (mercados campesinos).

- Los comerciantes de las plazas de mercado.

- Vendedores informales de los alrededores de las plazas de mercado.

- Tiendas de barrio.

- Consumidores de bienes y servicios ofrecidos por las plazas de mercado.

- Instituto para la Economía Social (IPES).

- Secretaría Distrital de Desarrollo Económico (SDDE).

- Secretaría Distrital de Salud (SDS).

- Secretaría Distrital de Integración Social (SDIS).

El fortalecimiento y desarrollo comercial de las plazas de mercado, constituye una oportunidad por la fuerte vigencia del mercado interno en Bogotá y la región central, el debate nacional agrario en el marco del proceso de paz, el desarrollo de la conciencia ciudadana sobre la diversidad y el medio ambiente y el patrimonio cultural inmaterial de la gastronomía criolla y los mercados campesinos (IPES, 2012).

De igual manera, desde la parte institucional, las plazas pueden dar respuesta a las demandas institucionales de alimentos; además estas son un eje entre las demandas de sujetos y circuitos de la economía popular en los territorios de la ciudad.

$\mathrm{Al}$ hacer una evaluación de resultados del proyecto "Fortalecimiento del sistema distrital de plazas de mercado", durante el cuatrienio (2012-2016), respecto a la programación y enfoque propuestos en su formulación y la gestión realizada con los actores de la cadena de comercialización de los productos de las plazas: productores campesinos, tenderos, consumidores, comerciantes, y su articulación con el sistema de abastecimiento en el marco de la política de seguridad alimentaria y nutricional para el distrito, y teniendo en cuenta la información secundaria registrada en los informes de gestión y de rendición de cuentas emitidos por el Instituto para la Economía Social y entrevistas con profesionales de apoyo del proyecto, se encuentra que: 
De acuerdo con el informe de gestión 2015 (Gómez, 2015) del Instituto para la Economía Social (IPES), los principales logros de gestión con respecto al proyecto en mención fueron:

- Se logró consolidar el posicionamiento de la cultura gastronómica de las plazas de mercado, mediante el fortalecimiento de las unidades productivas correspondientes a la actividad económica de restaurantes y venta de alimentos procesados, especialmente en las plazas de mercado La perseverancia, Doce de Octubre, 20 de Julio y Samper Mendoza, en donde se hizo énfasis en la implementación de buenas prácticas de manipulación de alimentos.

- Se lograron conformar y activar los comités de plaza y de desarrollo integral, en las 19 plazas de mercado distritales, con el fin de consolidar la gobernanza, gobernabilidad y participación de los comerciantes en su desarrollo y fortalecimiento.

- Se lograron mejorar y reforzar estructuralmente las plazas de mercado del Restrepo, 20 de Julio, Quirigua y Fontibón.

- Se tramitaron y radicaron ante la Secretaría Distrital de Planeación, la totalidad de los planes de regularización y manejo de las plazas distritales de mercado.

- Se lograron implementar planes de saneamiento básico en las 19 plazas de mercado distritales, en los cuales se encuentran incluidos los programas de gestión integral de residuos sólidos, control de vectores y limpieza y desinfección.

- Se logró implementar un plan de gestión integral de residuos orgánicos y su posterior aprovechamiento en cuatro plazas: Quirigua, Doce de Octubre, Siete de Agosto y Las Ferias, con el apoyo del convenio suscrito entre Fundases y la UAESP.

Si bien se identifican resultados de gestión importantes en cuanto al posicionamiento de la cultura gastronómica, estrategias de fortalecimiento empresarial, social y cultural, conformación y activación de los comités de plaza y de desarrollo integral, entre otros. También, se pueden evidenciar deficiencias en relación con la gestión de la política de seguridad alimentaria y nutricional, en cuanto a la articulación con las otras secretarías que son responsables de su ejecución, como la SDDE, SDIS y SDS, las cuales no se evidencian en los informes.

$\mathrm{Al}$ respecto se hicieron entrevistas con profesionales de apoyo (quienes se encargaban de fortalecer los procesos de mantenimiento, adecuación e infraestructura, gobernanza y gestión económica, en el cual el autor fue el encargado de hacer seguimiento de este último, durante el periodo 2012-2015) a la gestión de las plazas de mercado, estos manifestaron que desde que nació el Instituto para la Economía Social (IPES) en el 2007 y por ende, se le hizo entrega de la administración de las 19 plazas distritales de mercado, se ha formulado un proyecto para cada periodo de gobierno distrital (cuatrienio), para 
fortalecer y administrar las plazas, pero solo hasta este proyecto del plan de desarrollo Bogotá Humana (2012-2016), se ha tenido en cuenta el factor de la gestión económica, pensando no solo en la administración e infraestructura de las plazas, sino también en los comerciantes que aunque pagan un canon por el uso y aprovechamiento económico de los locales que usufructúan; también es necesario reactivar las dinámicas comerciales y propender por la seguridad alimentaria del Distrito.

Para este fin se han hecho rutas de emprendimiento y fortalecimiento comercial por medio de curso impartidos por el SENA sobre manipulación de alimentos, costos y presupuestos, entre otros, los cuales han originado que las plazas se reactiven y los comerciantes mejoren sus ingresos y por ende su calidad de vida, garantizando las dimensiones de la seguridad alimentaria: disponibilidad, acceso, utilización y estabilidad de los alimentos. Que según la FAO (2006) comprende:

Disponibilidad: la existencia de cantidades suficientes de alimentos de calidad adecuada, suministrados a través de la producción del país o de importaciones (comprendida la ayuda alimentaria).

Acceso: acceso de las personas a los recursos adecuados (recursos a los que se tiene derecho) para adquirir alimentos apropiados y una alimentación nutritiva. Estos derechos se definen como el conjunto de todos los grupos de productos sobre los cuales una persona puede tener dominio en virtud de acuerdos jurídicos, políticos, económicos y sociales de la comunidad en que vive (comprendidos los derechos tradicionales, como el acceso a los recursos colectivos).

Utilización: utilización biológica de los alimentos a través de una alimentación adecuada, agua potable, sanidad y atención médica, para lograr un estado de bienestar nutricional en el que se satisfagan todas las necesidades fisiológicas. Este concepto pone de relieve la importancia de los insumos no alimentarios en la seguridad alimentaria.

Estabilidad: para tener seguridad alimentaria, una población, un hogar o una persona deben tener acceso a alimentos adecuados en todo momento. No deben correr el riesgo de quedarse sin acceso a los alimentos a consecuencia de crisis repentinas (por ejemplo, una crisis económica o climática) ni de acontecimientos cíclicos (como la inseguridad alimentaria estacional). De esta manera, el concepto de estabilidad se refiere tanto a la dimensión de la disponibilidad como a la del acceso de la seguridad alimentaria. (p. 1)

\section{Inseguridad alimentaria en la localidad de Barrios Unidos}

Según la Cumbre Mundial sobre la Alimentación (1996):

Existe seguridad alimentaria cuando todas las personas tienen en todo momento acceso fisico y económico a suficientes alimentos inocuos y nutritivos para satisfacer sus necesidades 
alimenticias y sus preferencias en cuanto a los alimentos a fin de llevar una vida activa y sana. (p. 1)

Se considera que un hogar está en una situación de inseguridad alimentaria cuando sus miembros no disponen de manera sostenida de alimentos suficientes en cantidad y calidad, según las necesidades biológicas de estos. Por ende, se hace necesario incluir la dimensión de seguridad alimentaria en la evaluación del desarrollo humano, como un desarrollo por medio del crecimiento económico con equidad social, la transformación de los métodos de producción y de los patrones de consumo, que se sustentan en el equilibrio ecológico y soporte vital de una región. Teniendo como eje y corazón de este al ser humano (Brundtland, 1987).

Según Gallegos (2010), se debe incluir la dimensión de seguridad alimentaria en la evaluación del desarrollo humano por razones tales como:

- La seguridad alimentaria es fin y medio de desarrollo.

- En el mundo actual el hambre y la malnutrición se reconocen internacionalmente como uno de los grandes problemas que debe resolver la humanidad.

- A pesar de ello el hambre y la malnutrición continúan siendo un problema en gran parte silenciado.

- $\quad$ Responde a una demanda del PNUD.

- Es un instrumento de lucha contra el hambre y la malnutrición.

Por tal motivo, para este estudio es muy importante tener un conocimiento cercano a la realidad de Bogotá al respecto, y así poder definir si la localidad tiene deficiencia en la calidad y cantidad de alimentos que consumen. Por ende, se tomó como base la última "Encuesta distrital de demografía y salud Bogotá, 2011 ", para analizar la situación de la localidad de Barrios Unidos.

Según la encuesta, para la medición de la inseguridad alimentaria en el hogar (ISAH) se utiliza una escala compuesta por varios ítems, que cubren la preocupación de los hogares porque los alimentos se van a acabar y no se tienen los recursos para adquirirlos, la reducción de la cantidad de alimentos disponibles en el hogar, el deterioro de la calidad de la alimentación y la experiencia de hambre. En dicha encuesta algunas preguntas están dirigidas al hogar, otras preguntas se refieren a los adultos y a los niños que habitan en el domicilio (Profamilia, 2011). Los datos para Bogotá fueron los siguientes: 
Tabla 2. Inseguridad alimentaria de Bogotá por localidades

\begin{tabular}{|c|c|c|c|c|c|}
\hline Localidad & Seguro & $\begin{array}{l}\text { Inseguridad } \\
\text { alimentaria }\end{array}$ & $\begin{array}{c}\text { Inseguridad } \\
\text { leve }\end{array}$ & $\begin{array}{c}\text { Inseguridad } \\
\text { moderada }\end{array}$ & $\begin{array}{c}\text { Inseguridad } \\
\text { severa }\end{array}$ \\
\hline Usaquén & $87.7 \%$ & $12.3 \%$ & $8.4 \%$ & $3.7 \%$ & $0.2 \%$ \\
\hline Chapinero & $89.3 \%$ & $10.7 \%$ & $7.7 \%$ & $2.3 \%$ & $0.7 \%$ \\
\hline Santa Fe & $64.5 \%$ & $35.5 \%$ & $24.1 \%$ & $9.0 \%$ & $2.5 \%$ \\
\hline San Cristobal & $61.8 \%$ & $38.2 \%$ & $29.7 \%$ & $7.1 \%$ & $1.4 \%$ \\
\hline Usme & $59.6 \%$ & $40.4 \%$ & $29.7 \%$ & $9.4 \%$ & $1.3 \%$ \\
\hline Tunjuelito & $77.6 \%$ & $22.4 \%$ & $17.5 \%$ & $4.7 \%$ & $0.2 \%$ \\
\hline Bosa & $60.5 \%$ & $39.5 \%$ & $26.9 \%$ & $10.2 \%$ & $2.4 \%$ \\
\hline Kennedy & $77.3 \%$ & $22.7 \%$ & $17.6 \%$ & $4.0 \%$ & $1.0 \%$ \\
\hline Fontibón & $87.8 \%$ & $12.2 \%$ & $10.3 \%$ & $2.0 \%$ & $0.0 \%$ \\
\hline Engativa & $81.8 \%$ & $18.2 \%$ & $15.8 \%$ & $2.4 \%$ & $0.0 \%$ \\
\hline Suba & $79.8 \%$ & $20.2 \%$ & $15.0 \%$ & $4.3 \%$ & $0.9 \%$ \\
\hline Barrios Unidos & $82.0 \%$ & $18.0 \%$ & $13.7 \%$ & $3.8 \%$ & $0.5 \%$ \\
\hline Teusaquillo & $91.4 \%$ & $8.6 \%$ & $6.3 \%$ & $2.3 \%$ & $0.0 \%$ \\
\hline Mártires & $72.5 \%$ & $27.5 \%$ & $17.8 \%$ & $7.7 \%$ & $2.0 \%$ \\
\hline Antonio Nariño & $74.4 \%$ & $25.6 \%$ & $19.4 \%$ & $5.0 \%$ & $1.2 \%$ \\
\hline Puente Aranda & $78.3 \%$ & $21.7 \%$ & $18.5 \%$ & $2.4 \%$ & $0.8 \%$ \\
\hline Candelaría & $71.3 \%$ & $28.7 \%$ & $18.8 \%$ & $8.4 \%$ & $1.4 \%$ \\
\hline Rafael Uribe Uribe & $67.1 \%$ & $32.9 \%$ & $21.7 \%$ & $9.4 \%$ & $1.9 \%$ \\
\hline Guidad Bolívar & $64.2 \%$ & $35.8 \%$ & $26.8 \%$ & $8.5 \%$ & $0.5 \%$ \\
\hline Sumapaz & $45.2 \%$ & $54.8 \%$ & $47.8 \%$ & $6.5 \%$ & $0.4 \%$ \\
\hline
\end{tabular}

Fuente. Profamilia, SDP y SDIS. Encuesta distrital de demografia y salud Bogotá, 2011

En la tabla anterior se evidencia claramente el nivel de seguridad e inseguridad alimentaria por localidad, teniendo en cuenta 3 niveles para medirla, como lo son: inseguridad leve, moderada y severa.

El grado de inseguridad alimentaria en Bogotá para el 2011 se encontraba en un $24.3 \%$, en donde el $18.2 \%$ correspondía a la inseguridad alimentaria leve. 
El Plan de Desarrollo Bogotá Humana se propuso reducir a $5 \%$ el grado de inseguridad alimentaria moderada y severa de las familias, este indicador se encontraba en $6.1 \%$ de acuerdo CON la primera encuesta distrital de demografía y salud de Bogotá (EDDS) adelantada por Profamilia y la Alcaldía Mayor en el 2011.

Este mismo estudio hace claridad sobre las características de los grados de inseguridad alimentaria, que permiten identificar a la población que requiere inmediatamente apoyo alimentario.

$\mathrm{Al}$ respecto, a continuación se presenta el grado de inseguridad alimentaria en la localidad de Barrios Unidos en los tres niveles mencionados:

Figura 4. Grado de inseguridad alimentaria en la localidad de Barrios Unidos

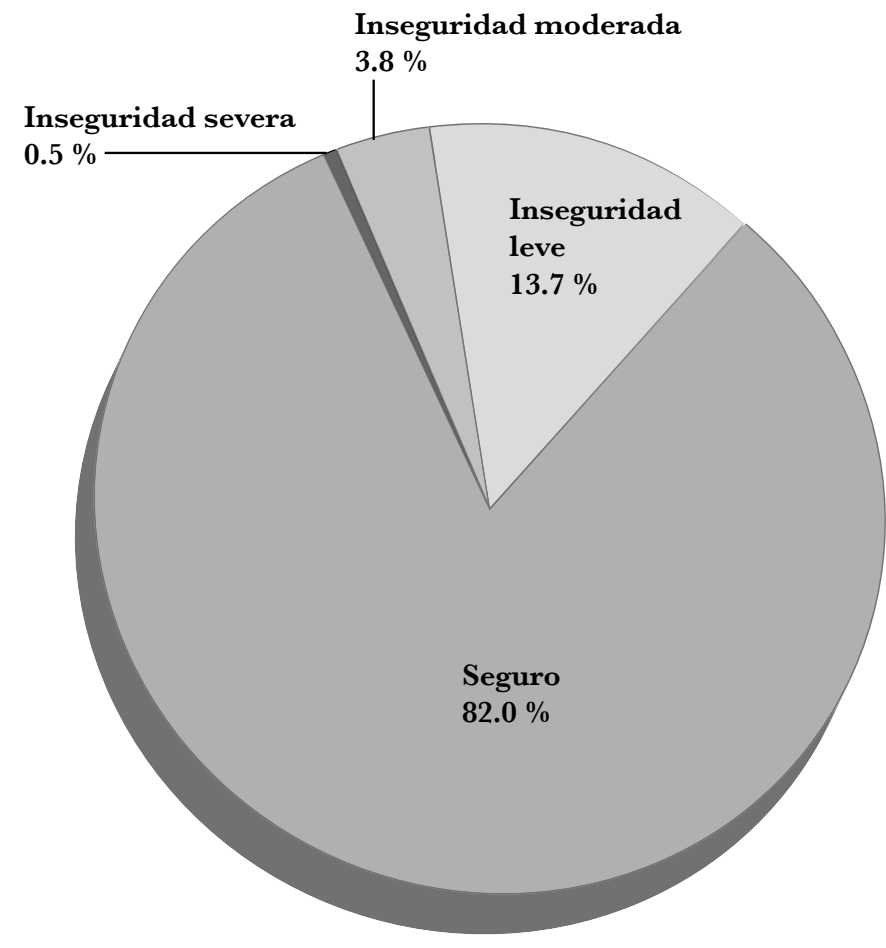

Fuente. Elaboración propia con base en Profamilia, SDP y SDIS. Encuesta distrital de demografía y salud Bogotá, 2011

En la localidad de Barrios Unidos el índice de inseguridad alimentaria para el año 2011 fue de $18 \%$, lo correspondiente a 13.688 hogares, distribuida así: leve $13.7 \%$ (10.418 hogares), moderada $3.8 \%$ (2.890 hogares) y severa $0.5 \%$ (380 hogares). En Barrios Unidos los índices de inseguridad alimentaria leve, moderada y severa fueron menores a las del Distrito Capital y Colombia. 
Con respecto a las plazas distritales de mercado que se encuentran en la localidad a la cual se hace referencia, quizás una solución para disminuir la inseguridad alimentaria sería la inclusión de los productores o cultivadores como un comerciante más de la plaza, es decir que no va a vender sus productos directamente a los comerciantes, sino que va a ocupar un puesto y va a vender sus productos directamente a los consumidores (Forero, 2003).

Esta venta directa ya que es realizada por el mismo productor, no solo ayudaría a bajar el precio del producto final (pues no tiene intermediarios), sino que también podría asegurar que el producto está en buenas condiciones para ser consumido, lo cual ayudaría a que las familias de la localidad tuvieran alimentos suficientes en cantidad y calidad según sus necesidades biológicas.

\section{Una medición del valor monetario del consumo de alimentos estimado para la localidad de Barrios Unidos}

Teniendo presente lo anterior, se denota que para el 2015 no hay cifras concretas, ni estudios realizados del consumo de alimentos a nivel monetario que sean recientes, por ende, como ejercicio investigativo de la maestría en Planeación para el Desarrollo, se propuso la elaboración de un modelo económico simple, para saber cuánto gastaron las familias en la compra de alimentos no procesados para su consumo en la localidad.

El modelo se basó a partir de los datos de la encuesta de calidad de vida en el año 2003 que se realizó en el gobierno de Luis Eduardo Garzón, con su plan de desarrollo Bogotá sin hambre. A partir de esta encuesta, el Instituto Latinoamericano de Servicios Legales Alternativos (ILSA), realizó una publicación en el 2005 denominada "Consumo de alimentos en Bogotá, déficit y canasta básica recomendada" (Yepes y Suárez, 2005), en donde se hizo un análisis exhaustivo por localidades y estratos del consumo de alimentos por valor monetario, cantidades físicas y canasta básica recomendada, entre otros.

Estás dos fuentes de información permitieron revisar los resultados y tener una idea cercana del comportamiento del consumo de alimentos a nivel monetario, de allí se planteó la creación de un modelo en donde se tuvieran en cuenta cambios tales como el incremento de la población y el incremento de los precios en la ciudad de Bogotá, entre el año 2004 y 2015, esto con el fin de tomar como referencia el estudio del 2003 y traerlo al valor presente (2015), que es la innovación y que adicional, permite a los tomadores de decisión información actual. 
Como se manifestó anteriormente, las variables y sus respectivos valores que fueron utilizados para estimar el valor del consumo de alimentos en la localidad son:

Tabla 3. Población total y consumo de alimentos en la localidad de Barrios Unidos (2003 y 2015)

\begin{tabular}{|ccccc|}
\hline Localidad & $\begin{array}{c}\text { Población 2003 } \\
\text { (habitantes) }\end{array}$ & $\begin{array}{c}\text { Población 2015 } \\
\text { (habitantes) }\end{array}$ & $\begin{array}{c}\text { Consumo } \\
\text { alimentos 2003 } \\
\text { (pesos) }\end{array}$ & $\begin{array}{c}\text { Consumo } \\
\text { fruver 2015 } \\
\text { (pesos) }\end{array}$ \\
\hline Barrios Unidos & 178.704 & 240.960 & $\$ 20.127 / 962.425$ & $\$ 2.779 .013 .790$ \\
\hline
\end{tabular}

Fuente. Departamento Administrativo Nacional de Estadística y Secretaria Distrital de Planeación. Bogotá, D. C. Proyecciones de población 2005-2015

Tabla 4. Inflación en Bogotá entre 2004 y 2015

\begin{tabular}{|cc|}
\hline \multicolumn{2}{|c|}{ Inflación Bogotá } \\
\hline Año & \% \\
\hline 2004 & 5.38 \\
\hline 2005 & 4.84 \\
\hline 2006 & 4.13 \\
\hline 2007 & 5.57 \\
\hline 2008 & 7.49 \\
\hline 2009 & 1.88 \\
\hline 2010 & 3.25 \\
\hline 2011 & 3.21 \\
\hline 2012 & 2.43 \\
\hline 2013 & 2.43 \\
\hline 2014 & 3.77 \\
\hline 2015 & 6.62 \\
\hline
\end{tabular}

Fuente. Departamento Administrativo Nacional de Estadística, índice de precios al consumidor por ciudad, serie histórica para rango de fechas dado, 2015 
Utilizando las variables de los cuadros anteriores se halló el consumo de alimentos estimado mensualmente para 2015 en la localidad, aplicando la siguiente fórmula:

Consumo alimentos (estimado 2015) = ECCP (2015) * ECIPC (2004-2015)

$\boldsymbol{E} \boldsymbol{C} \boldsymbol{C P}$ (2015): estimado crecimiento consumo con población de 2015

ECIPC (2004-2015): estimado crecimiento del IPC entre 2004 y 2015

Para hallar el estimado crecimiento consumo con población de 2015 se aplica la siguiente fórmula:

$\boldsymbol{E C C P}$ (2015) = (Población $2015 *$ Consumo alimentos 2003) / (Población 2003)

Para hallar el estimado crecimiento IPC entre 2004 y 2015 se aplica la siguiente fórmula:

$$
\begin{gathered}
\boldsymbol{E C I P C}(\mathbf{2 0 0 4}-\mathbf{2 0 1 5})=[1+(I P C 2004)] *[1+(I P C 2005)] *[1+(I P C \text { 2006 })] * \\
{[1+(I P C 2007)] *[1+(I P C 2008)] *[1+(I P C 2009)] *[1+(I P C 2010)] *[1+} \\
(I P C 2011)] *[1+(I P C 2012)] *[1+(I P C 2013)] *[1+(I P C 2014)] *[1+(I P C 2015)] \\
\text { ECIPC (2004- 2015) }=1.645262785
\end{gathered}
$$

Por lo tanto, el resultado para el consumo de alimentos a nivel general será el siguiente:

\section{Consumo alimentos (estimado 2015): \$44'652.498.880}

Para hallar el consumo de fruver estimado mensualmente para 2015 en la localidad se aplica la siguiente fórmula:

Consumo fruver (estimado 2015) = ECCFP (2015) *ECIPC (2004-2015)

$\boldsymbol{E C C F P ~ ( 2 0 1 5 ) : ~ e s t i m a d o ~ c r e c i m i e n t o ~ c o n s u m o ~ d e ~ f r u v e r ~ c o n ~ p o b l a c i o ́ n ~ d e ~} 2015$

ECIPC (2004 - 2015): Estimado crecimiento del IPC entre 2004 y 2015

Para hallar el estimado crecimiento de consumo de fruver con población de 2015 se aplica la siguiente fórmula:

$$
\boldsymbol{E C C P} \text { (2015) = (Población } 2015 * \text { Consumo fruver 2003) / (Población 2003) }
$$

El valor del IPC es constante y se tiene presente el mismo valor hallado anteriormente:

$$
\boldsymbol{E} \boldsymbol{C I P C}(\mathbf{2 0 0 4}-\mathbf{2 0 1 5})=1.645262785
$$

Por lo tanto, el resultado para el consumo de fruver será el siguiente:

Consumo fruver (estimado 2015): \$6'165.050.765 
La localidad de Barrios Unidos para diciembre de 2015 tenía una población aproximada de 240.960 habitantes, equivalente a 59.886 hogares (Secretaría Distrital de Planeación. Subsecretaria de Información y Estudios Estratégicos. Dirección de Información, Cartografía y Estadística. Inventario de Información en Materia Estadística sobre Bogotá. Proyecciones de Hogares y Viviendas 2006-2015). Por ende, cada hogar en promedio lo conforman 4 personas. Teniendo en cuenta lo anterior, y analizando los resultados del modelo propuesto, se puede afirmar que:

El gasto en consumo de alimentos de toda la localidad mensualmente para diciembre de 2015 en promedio fue de $\$ 44^{\prime} 652.498 .880$. Esto indica que el gasto promedio mensual por hogar fue de $\$ 745.625$, y por ende, por habitante fue de $\$ 186.406$.

$\mathrm{Al}$ mirar la especificidad del consumo, se toma como referencia el gasto de consumo en fruver mensualmente para diciembre de 2015, que fue de $\$ 6{ }^{\prime} 165.050 .765$. Esto indica que el gasto promedio mensual por hogar fue de $\$ 102.946$ y por habitante fue de $\$ 25.736$.

\section{Consideraciones finales}

Después de realizado el proceso de investigación y contraste tanto de las fuentes primarias, en especial, el modelo de consumo de alimentos y fuentes secundarias, se puede decir que el modelo de desarrollo o planificación de las plazas a partir del "Fortalecimiento del sistema distrital de plazas de mercado", se preocupó principalmente por la gobernanza, como ese elemento que buscaba fortalecer la administración y el manejo de las plazas desde lo institucional hacia estas, el mantenimiento, adecuación e infraestructura de la planta física de las plazas y la gestión económica que aunque intentó buscar estrategias para dinamizar la recuperación comercial en las 19 plazas distritales de mercado, no tuvo presente esas dinámicas comerciales entre el comerciante y los visitantes, y se olvidó (por parte del proyecto) de esas relaciones sociales que solo se dan en estos espacios de interacción.

Por consiguiente, en cuanto a la política pública de "Fortalecimiento del sistema distrital de plazas de mercado"; se busca principalmente que los comerciantes como personas naturales o jurídicas mediante la suscripción de un contrato de uso administrativo y aprovechamiento económico regulado con el IPES, adquieran el derecho de uso de un local, puesto, bodega o espacio en la plaza de mercado, para el abastecimiento de productos básicos de consumo doméstico, beneficiando tanto a la comunidad que los consume como a los mismos comerciantes, ya que les genera utilidades y contribuye a la seguridad alimentaria del sector donde se encuentran las plazas. 
En cuanto a las plazas distritales de mercado de la localidad de Barrios Unidos, se debe hacer una estrategia que las visibilice, con el fin de que las personas las visiten y así potenciarlas, no solo económicamente y de manera estructural o física, sino teniendo presente que las plazas cuentan sobre la historia y el crecimiento de la ciudad; puesto que son puntos de encuentro para discutir temas de política, deportes o economía; Además, allí aún sobreviven los mercados campesinos y esta es una forma directa de apoyar al campo; los campesinos de los municipios más cercanos se dedican diariamente a sembrar alimentos de calidad para traerlos a las ciudades y exhibirlos en estos lugares, se podría decir que es como pedir un domicilio del campo, pero sin intermediarios ni recargo.

En tanto, al analizar los precios de los productos ofrecidos en las plazas de mercado Siete de Agosto y Doce de Octubre, se evidencia que son económicos y asequibles a cualquier canasta familiar de cualquier estrato. Comprando en las plazas se ahorra dinero y además se apoya al campesino. Además de ser lugares en donde se venden por porciones y no existe la obligación de comprar por paquetes determinados y que hace parte de la tradición de ñapa (se usa normalmente cuando alguien va a la tienda, supermercado o plaza de mercado a comprar frutas, verduras o víveres a granel, y pide que se le dé una pequeña cantidad adicional gratuitamente). Entonces, hay una interacción directa con el comerciante que a la vez puede ser la persona que cultiva los alimentos, así que estos se pueden oler, tocar y hasta degustar antes de comprarlos. Están a la mano sin empaques ni vitrinas.

Basado en los resultados de la medición del valor monetario del consumo de alimentos, se puede inferir que teniendo presente que el gasto promedio mensual por hogar en la localidad de Barrios Unidos fue de $\$ 745.625$, este valor está por encima del valor del salario mínimo de dicho año (\$644.350). Esto puede significar que las personas que conforman cada hogar (en promedio 4 para esta localidad), en conjunto ganan por lo menos dos salarios mínimos (\$1’288.700). Es decir, gastan un $57.85 \%$ en alimentos no procesados.

Lo anterior también tiene su explicación en el nivel de vida de los habitantes de la localidad, pues según la Cámara de Comercio de Bogotá (2007) no existe el estrato 1 y 2. La distribución de los hogares por estratos es: estrato 3 representa el $50.7 \%$, estrato 4 el $38.9 \%$, estrato 5 el $5 \%$ y los predios no residenciales el $5.3 \%$. Además, la población de la localidad no se está alimentando de la mejor manera, pues de esos $\$ 745.625$ que invierten en alimentos, solo el $13.8 \%$ (\$102.946) es para fruver. Con lo cual se denota que, aunque los hogares cuentan con los recursos para alimentarse bien y tener una seguridad alimentaria digna, no lo hacen.

Es así que, a partir de la observación que se realizó en la localidad, se puede afirmar que "los fruver y tiendas de barrio, se están tomando la ciudad de Bogotá, se ha 
perdido esa cercanía con el campesinado y tradiciones, las relaciones de confianza entre el comerciante y el consumidor final, la "ñapa" o encime, el fiado, en fin toda relación directa con las personas que traen los alimentos de algún lugar del país para ponerlos en nuestras mesas" (apartes del trabajo de campo realizado en el año 2015 por el autor).

Por lo tanto, se puede afirmar que hay una relación entre el desarrollo humano y la inseguridad alimentaria, puesto que se puede analizar la dimensión de la seguridad alimentaria en la medición y evaluación del desarrollo humano. Y esto lo corroboran los datos obtenidos sobre la inseguridad alimentaria en la localidad de Barrios Unidos. No hay que olvidar que los alimentos que se venden en las plazas de mercado Siete de Agosto y Doce de Octubre, son saludables y naturales, garantizando así la seguridad alimentaria de las personas que compran sus víveres en estos mercados. Lo anterior, a pesar de que en la localidad hay amenazas tales como la tendencia de imposición de dietas corporativas, gracias a las propagandas radiales y televisivas y promociones de las grandes superficies, además del deterioro de los consumos alimentarios que se ven reflejados en los indicadores de inseguridad alimentaria.

Finalmente, los resultados de la investigación presentados en este artículo hacen un llamado para que exista información más actualizada para tomar decisiones en la política pública, en este caso particular del Instituto para la Economía Social (IPES), y sea a partir de datos reales y en valor presente lo que permita tomar estas decisiones. No hay que olvidar que toda política pública en pro del bienestar de la sociedad debe preocuparse por el "buen vivir"’ de la población que será sujeta de atención, y por ende, no se debe desconocer el impacto que tendrá esta en dicha población, para su desenvolvimiento social, económico, cultural y en general para su bienestar.

\section{¡Volvamos a las plazas de mercado!}

\section{¡Garanticemos la seguridad alimentaria de la población!}

\section{Referencias}

Brundtland, G. H. (1987). Informe Brundtland, nuestro futuro común.

Camara de Comercio de Bogotá. (2007). Perfil económico y empresarial de la localidad de Barrios Unidos. Bogotá.

2 Concebido como el espacio comunitario en donde existe reciprocidad, convivencia con la naturaleza, responsabilidad social y consenso (Houtart, 2011). 
Castiblanco, A. (2011). Las plazas de mercado como lugares de memoria en la ciudad: anclajes, pervivencias y luchas. Bogotá.

Correa, H. D. (2014). Las plazas tienen quien las quiera. Bogotá.

Decreto 315. (2006). Por el cual se adopta el Plan Maestro de Abastecimiento de alimentos y seguridad alimentaria para Bogotá Distrito Capitaly se dictan otras disposiciones. Bogotá.

Decreto 508. (2007). Por el cual se adopta la Política Pública de Seguridad Alimentaria y Nutricional para Bogotá, Distrito Capital, 2007-2015. Bogotá.

FAO. (2006). Informe de políticas de seguridad alimentaria, $n .^{\circ} 2$.

Forero, J. M. (Mayo, 2003). Mejoramiento operativo de una plaza de mercado distrital, Francisco de Paula Santander, Bogotá D. C. Bogotá, Colombia: Universidad de Los Andes.

Gallegos, A. (2010). La seguridad alimentaria mundial como indicador del desarrollo humano. Madrid: Universidad Politecnica de Madrid.

Gómez, C. (2015). Informe de gestión, Instituto para la Economia Social (IPES). Bogotá.

Hernández, R., Baptista, M. y Fernandez, C. (2010). Metodologia de la investigación. Mexico: McGraw-Hill / Interamericana Editores S. A. de C. V.

Houtart, F. (2011). El concepto de Sumak Kawsai (buen vivir) y su correspondencia con el bien común de la humanidad. ALAI, América Latina en Movimiento, 2.

Instituto Distrital de Turismo (IDT), Instituto para la Economia Social (IPES), y Fundación Escuela Taller (FET). (2013). Convenio de Asociacion entre IPES, IDT y FETB. Bogotá.

IPES. (2012). Proyecto 431 "Fortalecimiento del sistema distrital de plazas de mercado. Bogotá.

IPES. (2015). Sistema distrital de plazas de mercado. Instituto para la Economia Social (IPES). Recuperado de http:/ / www.ipes.gov.co/index.php/programas/plazas-de-mercado/ plaza-distrital-de-mercado-doce-de-octubre

Profamilia. (2011). Encuesta distrital de demografia y salud. Bogotá.

RAPE. (s.f.). Linea estrategica 5: soberania y seguridad alimentaria.

Russo, I. A. (2011). Mercados de abasto de los siglos XIX y XX de la plaza interior a no lugar.

Secretaría de Desarrollo Económico. (2012). Elementos de la apuesta de la política de soberania alimentaria en el plan de desarrollo Bogotá Humana. Bogotá. 
Revista CIFE / ISSN: 0124-3551 e-ISSN: 2248-4914 / Bogotá-Colombia / Vol. 20 N.o 32 / enero-junio 2018 / pp. $139-166$

Secretaría de Hacienda Distrital. (2015). Sistema de Presupuesto Distrital (Predis). Bogotá.

Whittingham, M. V. (2010). ¿Que es la gobernanza y para que sirve? Revista Analisis Internacional (RAI), 222.

Yepes, D. y Suárez, J. (2005). Consumo de alimentos en Bogotá, déficit y canasta básica recomendada. Bogotá: Instituto Latinoamericano de Servicios Legales Alternativos (ILSA). 